\title{
O Impacto da Judicialização dos Medicamentos no Estado do Mato Grosso
}

\author{
The Impact of Medicines Judicialization in the State of Mato Grosso
}

\author{
Ariadne de Melo Pereira; ${ }^{a}$ Mary Lúcia Ferraz Abrantesa; Marcelo Salles da Silva*b; Susana Nogueira Diniza; \\ Ivair Donizeti Gonçalves ; Regina Mara Silva Pereira ${ }^{\mathrm{a}}$; Márcio Luiz dos Santos*b \\ âniversidade Anhanguera São Paulo, Programa de Pós-Graduação Stricto Sensu em Farmácia. SP, Brasil. \\ 'Universidade Anhanguera São Paulo, Programa de Pós-Graduação Stricto Sensu em Biotecnologia e Inovação em Saúde. SP, Brasil. \\ *E-mail: marcelo.s.silva@anhanguera.com
}

\begin{abstract}
Resumo
A judicialização vem sendo utilizada como garantia de acesso a benefícios e serviços da saúde por intermédio do deferimento de ações judiciais, não observando as limitações do orçamento do poder público, o impacto financeiro e as características que essas ações pleiteadoras de medicamentos possam ocasionar para a coletividade. Este trabalho teve como objetivo, avaliar o perfil dos medicamentos requisitados por via judicial no Estado de Mato Grosso no período entre 2013 e 2014 e o total de ações e o volume de recursos gastos pela Secretaria de Estado de Mato Grosso no atendimento a estas ações, bem como o impacto da judicialização no orçamento público do Estado do Mato Grosso. A pesquisa demonstra uma habitual intervenção jurisdicional impondo a sobreposição do direito individual à saúde e assistência farmacêutica em relação às políticas públicas da saúde destinadas a coletividade. Para a realização deste trabalho, foram analisadas as informações de 6.547 mandados judiciais expedidos contra o Estado de Mato Grosso dentro deste período. Observou-se que o impacto no orçamento do estado de Mato Grosso representou aproximadamente $6 \%$ do orçamento da saúde, representando bloqueios judiciais no valor de R\$ 123.717.882.05, superando os valores pagos com medicamentos não judicializados. A estratégia adotada pela Secretaria de Saúde do Estado do Mato Grosso, para otimização de despesas com a judicialização dos medicamentos naquele Estado, demonstrou que reorganização administrativa é um instrumento eficaz aos estados na medida em que especializa os serviços de saúde e aumenta sua eficiência, diminuindo assim o volume de ações judiciais e consequentemente a oneração dos cofres públicos.
\end{abstract}

Palavras-chave: Judicialização. Saúde. Medicamentos.

\begin{abstract}
Judicialization has been used as a guarantee for health goods and services access through the granting of lawsuits, despite the limitations of government budget, the financial impact and characteristics that such actions seeking medication may cause to the community. Thus, this study aimed at analyzing drugs court ordered in Mato Grosso State during 2013 and 2014, and describe the drug profile, the total number of actions and the volume of resources spent by the Secretariat of Mato Grosso State, as well as the impact of judicialization on the public budget of Mato Grosso State. In order to carry out this work, the information of 6,547 court orders issued against the State of Mato Grosso within this period were analyzed. It was observed that the impact on the budget of Mato Grosso state represented approximately $6 \%$ of the total health budget, representing judicial blocks in the amount of $R \$ 123,717,882.05$, exceeding the amounts paid for non-legalized drugs. This research demonstrates a habitual jurisdictional intervention overlapping the individual right to health and pharmaceutical assistance in relation to the community public health policies. The strategy adopted by the Health Secretariat of Mato Grosso State to optimize expenses with the medicines judicialization demonstrated an administrative reorganization need as an effective instrument for the state in an attempt to specialize health services and increase their efficiency, leading to a decrease in lawsuits volume and consequently the burden on public coffers.
\end{abstract}

Abstract: Judicialization. Health. Medicines

\section{Introdução}

No Brasil, o Sistema Único de Saúde - SUS foi concebido como o conjunto de ações e serviços de saúde, criado pela Lei Orgânica da Saúde, após a entrada em vigor da Constituição Federal de 1988. O SUS é formado por atividades reunidas, prestadas por órgãos e instituições públicas federais, estaduais e municipais. Entre as principais atribuições do SUS está a formulação da política de medicamentos, equipamentos, imunobiológicos e outros insumos de interesse para a saúde e a participação na sua produção (BRASIL, 1990).

O SUS é considerado uma das maiores conquistas sociais e consagrado na constituição de 1988, garante acesso integral, universal e igualitário a qualquer pessoa aos serviços de saúde e fornecimento de medicamento, sendo financiado com recursos provenientes dos orçamentos da União, Estados, Municípios e do Distrito Federal, conforme previsão do artigo 195 da própria Carta Magna.

Os problemas advindos da qualidade ou efetividade dos serviços prestados pelo SUS têm permitido a propositura de inúmeras ações judiciais, ocasionando uma evidente intervenção do Poder Judiciário, nas atividades prestadas pelo Sistema Único de Saúde (PAIM et al, 2017). Tal intervenção é denominada judicialização da saúde.

A judicialização do acesso a medicamentos tem tomado grandes proporções (BARROSO, 2008), causando impactos significativos na estrutura, no financiamento e na organização do sistema de saúde do país (BOING et al, 2013). 
Segundo o Conselho Nacional de Justiça - CNJ, informações judiciais apontam para um crescimento acentuado de demandas judiciais envolvendo a saúde. No período de 2017, os tratamentos médico-hospitalares foram responsáveis pelo maior volume de processos judiciais, ou seja, 98.579 processos, tendo um aumento de $62 \%$ em relação ao ano de 2016. Nesse mesmo levantamento, foram destacados com maior demanda judicial, os tratamentos médico-hospitalares, fornecimento de medicamentos, saúde mental e planos de saúde (PAIXÃO, 2019)

Dados levantados pelo Conselho Nacional dos Secretários de Saúde - CONASS em 2003, já apontavam para as causas mais comuns de judicialização o Brasil. Naquele período, as causas mais comuns de ingresso das ações judiciais foram a falta do medicamento, a recusa para efetuar o fornecimento pelo fato da indicação estar em desacordo com os critérios estabelecidos no Protocolo Clínico e Diretrizes Terapêuticas - PCDT do Ministério da Saúde - MS e medicamento não padronizado e outros fatores como a solicitação de medicamentos não disponíveis no mercado nacional, normalmente com elevado custo de tratamento (BRASIL, 2004).

As ações judiciais são os maiores desafios que os gestores públicos enfrentam, entre outros fatores, geram individualização da demanda, em detrimento do coletivo. Várias propostas para solucionar ou minimizar as ações judiciais estão sendo discutidas pela Secretaria Estadual da Saúde - SES e pelo CONASS.

$\mathrm{O}$ estudo tem como objetivo avaliar o perfil dos medicamentos requisitados por via judicial no Estado de Mato Grosso no período entre 2013 e 2014 e o total de ações e o volume de recursos gastos pela Secretaria de Estado de Mato Grosso no atendimento a estas ações, bem como o impacto da judicialização no orçamento público do Estado do Mato Grosso.

A manutenção financeira do Sistema Único de Saúde envolve o comprometimento de verba pública, prevista no orçamento de todos os entes federativos, com previsão orçamentária e sujeitos a Lei de Responsabilidade Fiscal LRF (LENZA, 2019).

A Lei de Responsabilidade Fiscal tem uma organização bastante simples, estabelecendo etapas de controle das finanças públicas, que são: o planejamento, a transparência, o controle e a responsabilidade (BRASIL, 2000). Trata-se de uma instituição orçamentária disciplinadora das finanças públicas, que possibilita controlar os aspectos fiscais, o que permite, por sua vez, minimizar o efeito lesivo sobre o endividamento público. A promulgação da Lei de Responsabilidade Fiscal (LRF) é considerada o grande fator disciplinador das finanças públicas brasileiras, em todos os níveis (MACEDO, 2009).

A judicialização da saúde no Brasil, se por um lado é a atuação do Poder Judiciário na proteção de um direito constitucionalmente assegurado; por outro lado, tem uma vertente extremamente cruel, que é a intervenção do Poder Judiciário no agravamento das despesas públicas, em evidente desorganização da vida financeira governamental.

Estudo científico, ao analisar o impacto das liminares judiciais da saúde, nas contas públicas do estado de Santa Catarina no período de 2000 a 2006, Boing et al (2013), afirmam que $26,85 \%$ de todo o recurso destinado à aquisição de medicamentos no Estado foi utilizado no atendimento de ordens judiciais. Os autores ressaltam ainda, que equívocos judiciais na concessão de liminares para o fornecimento de medicamentos, comprometem severamente a gestão dos serviços de saúde do estado de Santa Catarina, trazendo sérias implicações na concretização das políticas públicas, pois dificultando a provisão e o planejamento dos recursos, gerando maiores dificuldades para a concretização de ações de saúde e fortalecimento do SUS.

\section{Desenvolvimento}

\subsection{Metodologia}

Os dados analisados foram coletados na Secretaria de Estado da Saúde do Estado do Mato Grosso, utilizando-se a base de dados dos mandados judiciais deferidos pelo Sistema Jurídico, arquivados na área do setor de Apoio Judicial, na Superintendência de Regulação, Coordenadoria de Controle e Avaliação, na área da Superintendência de Assistência Farmacêutica, Coordenadoria de Gestão de Assistência Farmacêutica - CAF, Superintendência de Orçamento.

Foram coletadas informações de 6.547 mandados judiciais expedidos contra o Estado de Mato Grosso entre os anos de 2013 e 2014, que contemplavam receitas com medicamentos, 3.047 mandados no ano de 2013 e 3.500 mandados referentes ao ano de 2014.

Esta pesquisa está de acordo com a Resolução 466/12 em relação aos riscos associados. Foi utilizado banco de dados anonimizados, em que não existe a possibilidade de identificar o indivíduo.

O acesso aos dados foi mediante assinatura da carta de anuência por parte da Superintendência de Assistência farmacêutica, Coordenadoria de Gestão de Assistência Farmacêutica (CAF) do Estado de Mato Grosso.

\subsection{A Judicialização da Saúde no Estado do Mato Grosso}

A judicialização da saúde é um problema que afeta todo o território federativo, provocando sistema de justiça exigindo avaliação de questões iminentemente técnica, vinculada a ciência da saúde. A ausência de conhecimento técnico na área da saúde, bem como de suporte à pareceres técnicos de saúde fornecido ao Poder Judiciário, tem estimulado a prolação de uma série de decisões judiciais equivocadas.

Diante desse quadro, no Estado do Mato Grosso foi criado o Núcleo de Atendimento Técnico (NAT) para oferecer suporte para os magistrados no momento de decidir demandas jurídicas relacionais à saúde. O NAT, foi criado em 19 de setembro de 2016, por força de um termo de cooperação técnica entre o Estado do Mato Grosso, representado pelo 
Tribunal de Justiça e a Secretaria de Saúde do Estado de Mato Grosso, para atender as Cidades de Cuiabá e Várzea Grande. Assim foi adotado um modelo de gestão da saúde pública, buscando um modelo mais eficiente.

\subsection{A Reorganização Administrativa da Secretaria de Saúde do Estado de Mato Grosso}

Em 2006, a Secretaria de Estado de Saúde começou a receber as primeiras decisões judiciais determinando o cumprimento das liminares de ações de assistência à saúde. Com a crescente demanda de liminares, em 2008 foi criado o "setor" de Apoio Judicial ligado à Superintendência de Regulação, Controle e Avaliação, sendo que este setor não está inserido no lotacionograma da Secretaria Estadual da Saúde do estado de Mato Grosso - SES/MT. Porém, em 04/10/2010 foi remodelado com a inserção de assessoria jurídica, divisão de responsabilidades entre a Coordenadoria de Assistência Farmacêutica e a Coordenadoria de Regulação vinculadas à Secretaria de Estado da Saúde do Mato Grosso.

No decorrer dos anos, essa problemática foi se agravando, chegando ao extremo e dispender grande parte do orçamento da SES para o custeio/compra de serviços/produtos para o cumprimento das liminares judiciais concedidas em processos que versaram sobre questões vinculadas à saúde.

O Estado do Mato Grosso é uma demonstração clara do impacto financeiro danoso advindos da judicialização desenfreada das questões de saúde no país. Para dar fluidez logística ao atendimento e cumprimento das inúmeras ordens judiciais, houve uma elevada e profunda reestruturação de departamentos na Secretaria de Saúde do Estado do Mato Grosso, criando setores que atuam desde o recebimento da ordem judicial, à setores que mapeiam os principais problemas, objeto de judicialização, para adoção de planos de ação administrativos obstando novas judicializações.

A reorganização administrativa da Secretaria de Estado da Saúde do Estado do Mato Grosso atendeu a Recomendação $n^{\circ}$ 31 de 30 de março de 2010 do Conselho Nacional de Justiça CNJ, que aconselhou os tribunais do país à adoção de medidas visando subsidiar os magistrados e demais operadores do direito, assegurando maior eficiência na solução das demandas judiciais envolvendo a assistência à saúde, evitando procrastinação no atendimento de decisões judiciais,

\section{a) Criação da Câmara Técnica}

A Câmara Técnica foi criada através da Portaria $n^{\circ}$ 001/2010/SES-MT/SMS-FUSC (Estado e Município), onde foi instituída uma Equipe Multiprofissional com a finalidade de dar suporte técnico, avaliar e subsidiar o Poder Judiciário e órgãos auxiliares com informações técnicas aos processos judiciais. Dentre outros, a atuação da Equipe Multidisciplinar atuar tecnicamente em processos judiciais, nas Promotorias de Justiça, nas Comarcas de Justiça daquele Estado, nas Auditorias e Ouvidorias do Estado de Mato Grosso.

A Câmara Técnica atua em um sistema de recebimentos de demandas judiciais, oferecendo suporte técnico ao Poder Judiciário. As análises técnicas realizadas nas ações judiciais permitiram verificar que, os serviços habilitados e credenciados pelo SUS não estão atendendo suficientemente as necessidades da população, gerando demandas reprimidas e longas filas de espera.

\section{b) Estrutura de Apoio Judicial/SURCA/SES}

Com o funcionamento das Câmara Técnica no Estado do Mato Grosso, também desencadeou a necessidade de uma melhor adequação da estrutura administrativa da Secretaria de Saúde do Estado do Mato Grosso, para dar atendimento às ordens judiciais.

Dessa forma, por intermédio da Portaria 102/2013/GBSES de 15 de agosto de 2013, ocorreu uma estruturação do Setor de Apoio Judicial no Estado do Mato Grosso, objetivando realizar levantamento de todos os Processos Judiciais e Recomendações Ministeriais pendentes de cumprimento e/ou resposta; fazer o diagnóstico e encaminhamento dos processos que não estão sendo cumpridos nos prazos estabelecidos e recomendar fluxos, visando dar agilidade ao cumprimento dos Processos Judiciais e Recomendações Ministeriais no recebimento de notificações e intimações inerentes a medicamentos, suplementos nutricionais, materiais médico-hospitalares, procedimentos médicos, hospitalares e laboratoriais.

\section{c) Assessoria de Demandas Judiciais}

No Estado do Mato Grosso, além de Setores de Apoio, houve a criação de uma Assessoria de Demandas Judiciais (ASSEJUD) na Secretaria de Estado de Saúde, subordinada e vinculada ao Gabinete do Secretário de Estado de Saúde, instituída através da Portaria $n^{\circ}$ 55/2015/GBSES/SES - art. $1^{\circ}$, como estratégia para gerenciar as demandas judiciais. Sendo o órgão estratégico da Secretaria de Estado de Saúde responsável por coordenar, monitorar, supervisionar, impulsionar e dar suporte, até o efetivo cumprimento pelas pastas judiciais.

Com a criação da ASSEJUD, unificaram-se todas as entradas para expedientes judiciais em um único setor, a fim de manter maior controle da demanda. Além de disponibilizar estatísticas analíticas das demandas para a utilização em instrumento de gestão, visando minimizar as dificuldades da população no acesso à saúde. A ASSEJUD também estreita o trâmite dos processos judiciais, tornando o cumprimento das ordens judiciais mais rápidas e com menor margem de erro.

A reestruturação administrativa do Estado do Mato Grosso possibilitou o aumento da resolutividade da rede de atenção, evitando o aumento de demandas judiciais por serviços de saúde e reduzir o custo dos atendimentos pelo planejamento prévio da oferta de serviços. Tudo isso, com o intuito de ajustar a oferta assistencial disponível no estado às necessidades do cidadão, evitando assim uma desorganização na vida financeira e orçamentária do sistema de saúde, por imposição de ordens judiciais. 


\subsection{Resultados e Discussão}

O volume de demandas Judiciais recebidas em desfavor da Secretária de Saúde de Mato Grosso, apontam para o fenômeno da judicialização da saúde e causam um forte impacto financeiro para a gestão, pois gera individualização da demanda em detrimento do coletivo. Atualmente, a judicialização está entre os maiores problemas das Secretarias de Saúde dos Estados, que enfrentam o crescente número de ações judiciais referente à saúde. As mesmas possuem orçamentos comprometidos e uma grande interferência do poder judiciário frente ao executivo, entre outros.

A judicialização do acesso ao medicamento tem tomado grandes proporções, causando impactos significativos na estrutura, no financiamento e na organização do sistema de saúde. Iniciou-se com ações que exigiam o fornecimento de medicamentos para o tratamento da AIDS, estratégia bemsucedida que se estendeu, para as mais variadas patologias (SANT'ANA, 2011).

Figura 1 - Liminares judiciais em processos envolvendo fornecimento de medicamentos no período de 2012-2014 no estado do Mato Grosso

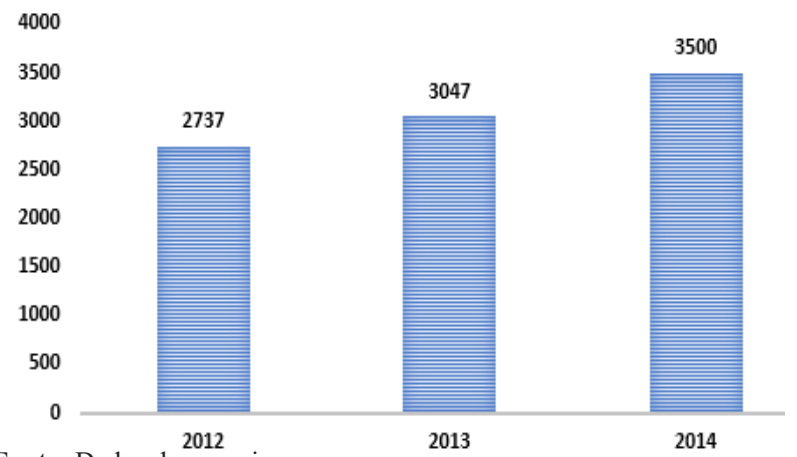

Fonte: Dados da pesquisa.

Podemos observar o crescente número de demandas judiciais a cada ano em Mato Grosso, apontam crescimento das despesas para atendimento e fornecimento de materiais de consumo e serviços de saúde para estas ações. Em 2013 houve crescimento de $11,33 \%$ em relação ao ano de 2012 , em 2014 o aumento alcançou 14,86\% em relação ao ano de 2013. Portanto, podemos observar que em apenas dois anos, o crescimento foi de aproximadamente $25 \%$.

Figura 2 - Ações judiciais envolvendo fornecimento de medicamento no estado de Mato Grosso - Distribuição por Comarca

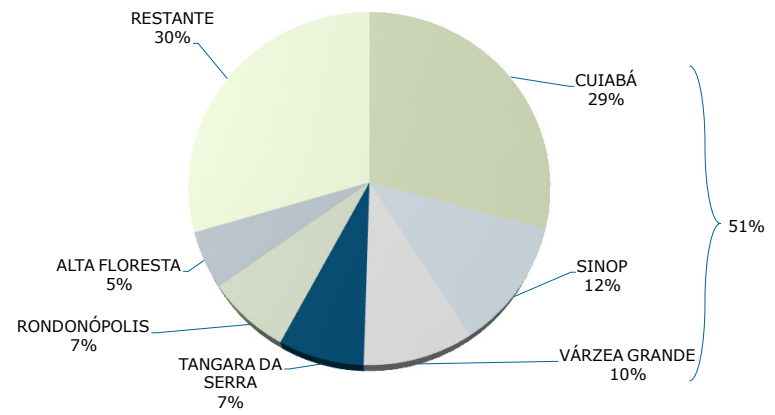

Fonte: Dados da pesquisa.
A distribuição por comarca no estado de Mato Grosso, possuindo 141 municípios e conforme Figura 2, a maioria das demandas se origina do município de Cuiabá, capital do Estado com 29\% da demanda, com população de 530.308 . Em segundo lugar a comarca de Sinop com $12 \%$ e população de 111.643, quarto município mais populoso do estado. Em terceiro lugar a comarca de Várzea Grande, equivalendo a $10 \%$ da demanda, com uma população de 2.954 .625 pessoas, de acordo com o censo IBGE (2010).

Figura 3 - Ações judiciais envolvendo fornecimento de medicamento no Estado de Mato Grosso. Distribuição por objeto da ação/especialidade

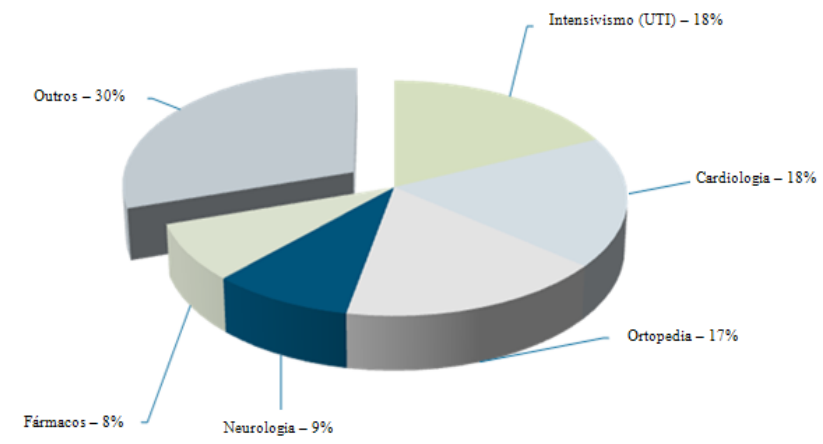

Fonte: Dados da pesquisa.

Considerando o levantamento efetuado pelo CONASS, as causas mais comuns de ingresso das ações judiciais foram à falta do medicamento (citada por $10 \mathrm{SES}$ ); recusa para efetuar o fornecimento, devido a indicação estar em desacordo com os critérios estabelecidos pelo Protocolo Clínico e Diretrizes Terapêuticas - PCDT do Ministério da Saúde (citada por 15 SES) e medicamentos não padronizado (citada por $16 \mathrm{SES}$ ). Outros fatores foram mencionados por quatro SES, entre eles, a solicitação de medicamentos não disponíveis no mercado nacional, normalmente, como elevado custo de tratamento (BRASIL, 2004).

A judicialização tornou-se recorrente no Brasil com decisões judiciais beneficiando qualquer tipo de tratamento e trazendo como consequencias as demandas individuais em detrimento das coletivas.

Figura 4 - Ações judiciais envolvendo fornecimento de medicamento no estado de Mato Grosso - Distribuição por objeto/especificidade

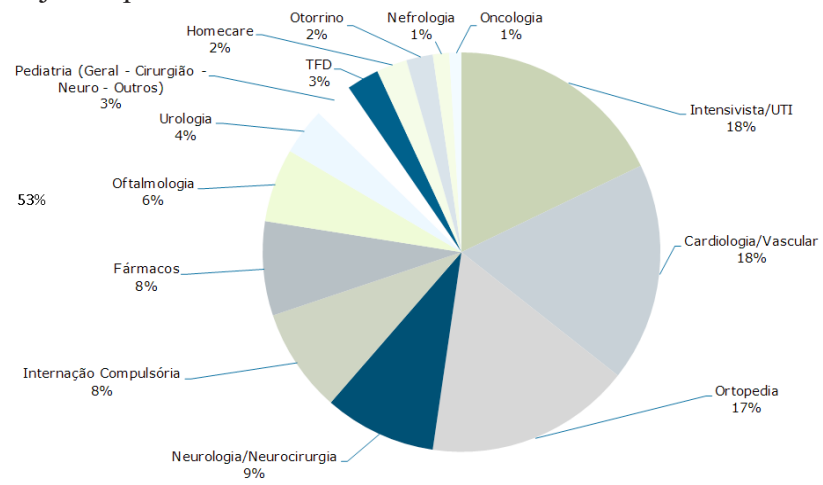

Fonte: Dados da pesquisa. 
Conforme a demonstração por especificidade, referente a judicialização no estado de Mato Grosso, observou-se que $18 \%$ referia-se a problemas de cardiologia/vascular, $18 \%$ intensivista/UTI, $17 \%$ ortopedia, 9\% neurologia/ neurocirurgia, $8 \%$ internação compulsória, $8 \%$ fármacos, $6 \%$ oftalmologia, 4\% urologia, 3\% pediatria (geral- cirurgia), $3 \%$ outros, 3\% TFD, 2\% homecare (cuidados domiciliares), 2\% otorrino, $1 \%$ nefrologia e $1 \%$ oncologia.

A implantação da decisão judicial, apresentou-se como uma das responsáveis pela desorganização do fluxo normal de atendimento dos pacientes. Através do seu caráter impositivo nas decisões judiciais, favoreceu pacientes beneficiados ao acesso de serviço de saúde sem considerar apenas critérios médicos, os quais as instâncias de gestão são obrigadas a cumpri-las, independentes da situação em que se apresenta sua oferta de serviços, sob pena de incorrerem em crime de desobediência à ordem judicial (BRASIL, 1940).

Segundo o Tribunal de Contas do estado do Mato Grosso (TCE-MT) em face dessa realidade, os pacientes buscam o Poder Judiciário para obter acesso aos serviços de saúde, pois a oferta é insuficiente. Aliado a isso, a baixa resolutividade da rede de atenção e o planejamento deficiente por parte do estado e dos municípios, também tem contribuído para o aumento da judicialização nos últimos anos.

Conforme dados das Planilhas de Controle de Liminares de Medicamentos, encaminhadas pelo Apoio Judicial da Coordenadoria de Assistência Farmacêutica da SES, memorando $\quad \mathrm{n}^{\mathrm{o}} \quad 0183 / 2014 / \mathrm{SAF} / \mathrm{CAF}-\mathrm{GEBEX} / \mathrm{SES}-$ MT, fls. 69 a 136, das autuações realizadas pela SES em 2014, aproximadamente 1.579 foram de solicitações de medicamentos.

Observou-se que dentre as demandas judiciais deferidas referentes a medicamentos, os itens mais solicitados foram Suplemento Alimentar, seguido pelos Anticonvulsionantes, Antidepressivos e Medicamentos Oncológico, de acordo com a classificação da ATC (Anatomical Therapeutic Chemical Code).

Quadro 1 - Bloqueios Judiciais no estado do Mato Grosso período 2013

\begin{tabular}{|c|c|}
\hline Bloqueio por Conta Corrente & R\$ Valor \\
\hline $\begin{array}{l}\text { Boqueio Judicial nas Contas FES Fonte } 112 \\
\text { (Recurso o Ministério da Saúde) e Fonte } 161 \\
\text { (Recursos de Convênios) }\end{array}$ & $9.495 .760,01$ \\
\hline $\begin{array}{l}\text { Bloqueio Judicial na Conta Única do Estado } \\
\text { Fonte } 134 \text { (Recursos do Tesouro Estadual) }\end{array}$ & $47.363 .108,87$ \\
\hline $\begin{array}{lc}\text { Total RS } \\
\end{array}$ & $56.858 .868,88$ \\
\hline
\end{tabular}

Fonte: Adaptado de Mato Grosso (2015).

O Quadro 2 demonstra que no ano de 2014 houve crescimento de $21,21 \%$ do volume financeiro bloqueado em ações judiciais no estado do Mato Grosso, em comparação aos bloqueios que ocorrerão no ano anterior.
Quadro 2- Volume financeiro dos bloqueios Judiciais no estado do Mato Grosso - período 2014

\begin{tabular}{|l|c|}
\hline \multicolumn{1}{|c|}{ Bloqueio por Conta Corrente } & \multicolumn{1}{c|}{ R\$ Valor } \\
\hline $\begin{array}{l}\text { Boqueio Judicial nas Contas FES Fonte } \\
112 \text { (Recurso o Ministério da Saúde) }\end{array}$ & $14.235 .296,62$ \\
\hline $\begin{array}{l}\text { Bloqueio Judicial nas Conta FES Fonte } \\
161 \text { (Recursos de Convênios) }\end{array}$ & $5.091 .417,71$ \\
\hline $\begin{array}{l}\text { Bloqueio Judicial na Conta Única do } \\
\text { Estado Fonte 134 (Recursos do Tesouro } \\
\text { Estadual) }\end{array}$ & $49.594 .442,19$ \\
\hline \multicolumn{1}{|c|}{ Total R\$ } & $68.921 .156,52$ \\
\hline
\end{tabular}

Fonte: Adaptado de Mato Grosso (2015).

A análise dos Quadros 1 e 2, evidencia que no ano de 2013 o valor R\$56.858.868,88 e no ano de 2014 o valor $\mathrm{R} \$$ 68.921.156,52, foram destinados ao atendimento por Bloqueio Judicial.

\section{Conclusão}

A quantidade de liminares com entrada na Secretaria de Saúde do Mato Grosso é crescente e constante, causando impacto significativo na estrutura, no financiamento e na sua organização. Demonstrando fragilidade na sua organização administrativa.

O pagamento dos programas da Secretaria Estadual de Saúde (SES) do Estado do Mato Grosso, que utilizou as subfunções do Governo, por ocasião dos Bloqueios Judiciais advindos da judicialização de questões relacionados à saúde, representou aproximadamente $6 \%$ (seis por cento) do orçamento estadual, destinado à pasta da Saúde; e superou os valores pagos com medicamentos não judicializados. Mas não é só, a fragilidade da organização administrativa exigiu um investimento público na criação de setores especializados para atender as demandas judiciais.

$\mathrm{O}$ desenvolvimento da pesquisa demonstrou ainda, que os problemas cardiovasculares e os intensivistas (UTI) são as especificidades com maior demanda judicial no estado de Mato Grosso (18\%), seguida de problemas ortopédicos (17\%), neurologia/neurocirurgia (9\%), internação compulsória (8\%), compras de fármacos $(8 \%)$, problemas oftalmológicos $(6 \%)$, urológicos $(4 \%)$, pediátricos $(3 \%)$, outros $(10 \%)$.

Observou-se ainda, que a maior demanda se concentrava no município de Cuiabá (29\%), seguida pela comarca de Sinop (12\%) e em terceiro lugar a comarca de Várzea Grande (10\%). Desta forma, verifica-se o fenômeno da sobreposição do direito individual a saúde e assistência farmacêutica em relação às políticas públicas da saúde destinadas a coletividade.

A interferência do Poder Judiciário no exercício das atividades do gestor público, que por intermédio de decisões judiciais oneram os gastos públicos de um orçamento em curso, gera um problema para o planejamento orçamentário e cumprimento das metas financeiras do Estado do Mato Grosso, em especial para atender as políticas públicas de saúde. 


\section{Referências}

BARROSO, L.R. Da falta de efetividade à judicialização excessiva: direito à saúde, fornecimento gratuito de medicamentos e parâmetros para a atuação judicial. UNIJUS, v.11, n.15, p.1338,2008 .

BRASIL. Supremo Tribunal Federal. ADFP (Arguição de Descumprimento de Preceito Fundamental). Relator: Ministro Celso de Mello. Diário Oficial da União (DOU) 04/05/2004, pág. 00012. Argte: Partido da Social Democracia Brasileira - PSDB: Argdo: Presidente da República. Brasília, DF. Disponível em: http://sbdp.org.br/arquivos/material/343_204\%20ADPF\%20 2045.pdf

BRASIL. Conselho Nacional de Secretários de Saúde. Recursos humanos: um desafio do SUS/Conselho Nacional de Secretários de Saúde. Brasília: CONASS, 2004 (Serie CONASS documento, v.5).

BRASIL. Constituição da República Federativa do Brasil. Diário Oficial da União - Seção 1, Brasília, DF, página 1, 5 out 1988.

BRASIL. Decreto Lei No 2.848, de 7 de dezembro de 1940. Código Penal. Diário Oficial da União - Seção 1, Brasília, DF, página 23911, 31 dez1940

BRASIL. Lei no 8.080, de 19 de setembro de 1990. Dispõe sobre as condições para a promoção, proteção e recuperação da saúde, a organização e o funcionamento dos serviços correspondentes e dá outras providências. Diário Oficial da União - Seção 1 - Brasília, DF, página18055, 20set1990

BRASIL. Lei Complementar $n^{\circ} 101$, de 4 de maio de 2000. Estabelece normas de finanças públicas voltadas para a responsabilidade na gestão fiscal e dá outras providências. Diário Oficial da União - Seção 1 - Brasília, DF, página 46, 05mai2000

BRASIL. Lei Complementar $\mathrm{n}^{\circ} 141$, de 13 de janeiro de 2012. Regulamenta o parágrafo $3^{\circ}$ do art. 198 da Constituição Federal para dispor sobre os valores mínimos a serem aplicados anualmente pela União, Estados, Distrito Federal e Municípios em ações e serviços públicos de saúde; estabelece os critérios de rateio dos recursos de transferências para a saúde e as normas de fiscalização, avaliação e controle das despesas com saúde nas 3 (três) esferas de governo; revoga dispositivos das Leis nos 8.080, de 19 de setembro de 1990, e 8.689, de 27 de julho de 1993; e dá outras providências. Diário Oficial da União - Seção 1 - Brasília, DF, página 1, 16jan2012

BRASIL. Portaria SNVS n ${ }^{\circ} 344$, de 12 de maio de 1998. Aprova o Regulamento Técnico sobre substâncias e medicamentos sujeitos a controle especial. Republicada no Diário Oficial da União, Brasília, DF, 1 fev.1999.

BOING, A. et al. A judicialização do acesso aos medicamentos em Santa Catarina: um desafio para a gestão do sistema de saúde. Rev. Direito Sanitário, v.14, n.1, p.82-97, 2013. doi:10.11606/ issn.2316-9044.v14i1p82-97

IBGE - Instituto Brasileiro de Geografia e Estatística. Censo Demográfico 2010. Características da população e dos domicílios: resultados do universo. Rio de Janeiro: IBGE, 2010.

LENZA, P. Direito Constitucional esquematizado. São Paulo: Saraiva, 2019.

MACEDO,J.J.;CORBARI,E.C.EfeitosdaLeideResponsabilidade Fiscal no endividamento dos municípios brasileiros: uma análise de dados em painéis. Rev. Contab. Finanç., v.20, n.51, 2009. doi: 10.1590/S1519-70772009000300004

MATO GROSSO. Secretaria Adjunta de Gestão Estratégica - Secretaria de Estado de Saúde de Mato Grosso. Relatório Referente à Judicialização da Saúde. Cuiabá: Secretaria Adjunta de Gestão Estratégica, 2015.

PAIM, L.F.N.A. et al. Qual é o custo da prescrição pelo nome de marca na judicialização do acesso aos medicamentos? Cad. Saúde Coletiva, v.25, n.2, p.201-209, 2017. doi:10.1590/1414$462 \times 201700020022$.

PAIXÃO, A.L.S. Reflexões sobre a judicialização do direito à saúde e suas implicações no SUS. Ciênc. Saúde Coletiva, v.24, n.6, 2019. doi: 10.1590/1413-81232018246.08212019

SANT'ANA, J.M.B.; PEPE, V.L.E. Essencialidade e assistência farmacêutica: considerações sobre o acesso a medicamentos mediante ações judiciais no Brasil. Rev. Panam. Salud Publica, v.29, n.2, 2011.

WERNECK VIANNA, L. et al. A judicialização da política e das relações sociais no Brasil. Rio de Janeiro: Revan, 1999. 